\title{
Ergodic problem \\ for the Hamilton-Jacobi-Bellman equation. I. Existence of the ergodic attractor
}

by

\author{
Mariko ARISAWA
}

CEREMADE, Université Paris-Dauphine, Place de Lattre de Tassigny, 75775 Paris Cedex 16.

ABSTRACT. - The problem of the convergence of the terms $\lambda u_{\lambda}(x)$, $\neq u(x, T)$ in the Hamilton-Jacobi-Bellman equations (HJBs) as $\lambda$ tends to $+0, T$ goes to $+\infty$, to the unique number is called the ergodic problem of the HJBs. We show in this paper what kind of qualitative properties exist behind this kind of convergence. The existence of the ergodic attractor is shown in Theorems 1 and 2 . Our solutions of HJBs satisfy the equations in the viscosity solutions sense.

RÉSUMÉ. - Le problème de la convergence des termes $\lambda u_{\lambda}(x), \frac{1}{T} u(x, T)$ dans les équations de Hamilton-Jacobi-Bellman (HJBs) quand $\lambda$ tends vers $+0, T$ tends vers $+\infty$, vers le numéro unique s'appelle le problème ergodique des HJBs. Nous montrons ici les propriétés qualitatives qui existent derrière ce type de convergence. L'existence de l'attracteur ergodique est démontrée dans les Théorèmes 1 et 2 . Nos solutions des HJBs satisfont les équations au sens de la solution de la viscosité.

\section{INTRODUCTION}

In this paper and in the subsequent papers of this series, we study the so-called ergodic problem of the Hamilton-Jacobi-Bellman equation (H.J.B. in short). We concern solutions of one of the following problems. 
(Stationary problem - infinite horizon control problem)

(1) $\sup _{\alpha \in A}\left\{-<b(x \cdot \alpha) \cdot \nabla u_{\lambda}(x)>+\lambda u_{\lambda}(x)-f(x, \alpha)\right\}=0, \quad x \in \Omega$,

(Time dependent problem - finite horizon control problem)

$$
\begin{gathered}
\frac{\partial u}{\partial t}(x, t)+\sup _{\alpha \in A}\{-<b(x, \alpha), \nabla u(x, t)>-f(x, \alpha)\}=0, \\
x \in \Omega, t>0, \\
u(x, 0)=0, \quad x \in \Omega .
\end{gathered}
$$

The goal is to study the convergence of terms $\lambda u_{\lambda}(x)$ in (1) and $\frac{1}{T} u(x, T)$ in (2) as $\lambda$ goes to 0 and $T$ goes to $+\infty$ respectively. Here, $\Omega$ is a bounded connected open smooth set in $\mathbb{R}^{n}$ (or the $n$ dimensional smooth manifold; $u_{\lambda}(x),(\lambda>0)$ and $u(x, t)$ are real-valued unknown functions defined in $\bar{\Omega}, \bar{\Omega} \times[0, \infty)$ respectively; $A$ is a metric set corresponding to the values of the controls for the underlying controlled dynamical system; $b(x, \alpha)$ is a continuous function on $\bar{\Omega} \times A$ with values in $\mathbb{R}^{n}$ which is Lipschitz continuous in $x$ uniformly in $\alpha ; f(x, \alpha)$ is a bounded continuous on $\bar{\Omega} \times A$ with values in $\mathbb{R}$. And we consider either one of the following boundary conditions: for the equation (1).

\section{(Periodic B.C.)}

$\bar{\Omega}$ is assumed to be a $n$ dimensional torus

$$
\mathbf{T}^{n}=\mathbb{R}^{n} / \prod_{i=1}^{n}\left(T_{i} \mathbf{Z}\right) \approx \prod_{i=1}^{n}\left[0, T_{i}\right],
$$

where $T_{i} \quad(1 \leq i \leq n)$ are real numbers and that $b(x, \alpha), f(x, \alpha)$ are periodic in $x_{i} \quad(1 \leq i \leq n)$ with the period $T_{i} \quad(1 \leq i \leq n)$.

(Neumann type B.C.)

$$
<\nabla u_{\lambda}(x), \gamma(x)>=0 \quad \text { on } \quad \partial \Omega
$$

(State constraints B.C.)

$$
u_{\lambda}(x) \text { is a viscosity supersolution of (1) in } \bar{\Omega}
$$


and for the equation (2), we impose (3) or either one of

(Neumann type B.C.)

$$
<\nabla u(x, t), \gamma(x)>=0 \quad \text { on } \quad \partial \Omega
$$

\section{(State constraints B.C.)}

$$
u(x, t) \text { is a viscosity supersolution of (2) in } \bar{\Omega} \times[0,+\infty),
$$

where $\gamma(x)$ is a smooth vector field on $\partial \Omega$ pointing outward i.e. denoting $n(x)$ the unit outward normal at $x \in \partial \Omega, \gamma(x)$ satisfies

$$
\exists \nu>0, \forall x \in \partial \Omega, \quad<n(x), \gamma(x)>\geq \nu
$$

Existence and uniqueness results for the H.J.B. equations (1), (2) with the boundary conditions stated above have been obtained in the viscosity solutions framework, the references of which we shall give at the end of the introduction. Now, assuming that the equations have a unique viscosity solution (the viscosity solution theory was introduced by M.G. Crandall and P.L. Lions in [7] to treat the nonlinear P.D.Es. in a generalized solutions framework), we shall mainly be concerned in this introduction with the following two issues: first, we want to explain why the convergence problem of $\lim _{\lambda \rightarrow 0} \lambda u_{\lambda}(x), \lim _{T \rightarrow \infty} \frac{1}{T} u(x, T)$ is called the "ergodic problem"; secondly, we state our main result namely the existence of a subset of $\bar{\Omega}$ which plays the role of an attractor for the control problem, we shall call this set the ergodic attractor.

First, we shall briefly mention the relationship between the convergence of $\lim _{\lambda \rightarrow 0} \lambda u_{\lambda}(x), \lim _{T \rightarrow \infty} \frac{1}{T} u(x, T)$ and the notion of "ergodicity" in the dynamical systems theory. For this, let us remind that the equations (1), (2) corresponds to the deterministic controlled dynamical system given by the following ordinary differential equations (9) (in the cases of periodic B.C. and state constraints B.C.) and (10) (in the case of Neumann type B.C.), and that the value functions $u_{\lambda}(x), u(x, t)$ given in (11), (12) below are the solutions of $(1),(2)$ respectively. The O.D.Es. are

$$
\begin{cases}\frac{d}{d t} x_{\alpha}(t)=b\left(x_{\alpha}(t), \alpha(t)\right) & \forall t \in \mathbb{R}, \\ x_{\alpha}(0)=x & x \in \bar{\Omega}, \forall \alpha \text { control, } \\ x_{\alpha}(t) \in \bar{\Omega} & \forall t \in \mathbb{R}, \forall \alpha \text { control, }\end{cases}
$$


and

$$
\left\{\begin{array}{l}
x_{\alpha}(t)=x+\int_{0}^{t} b\left(x_{\alpha}(s), \alpha(s)\right) d s-\int_{0}^{t} \gamma\left(x_{\alpha}(s)\right) d \beta_{s} \quad \forall t \in \mathbb{R}, \\
x_{\alpha}(0)=x \quad x \in \bar{\Omega}, \forall \alpha \text { control, } \\
x_{\alpha}(t) \in \bar{\Omega} \quad \forall t \in \mathbf{R}, \forall \alpha \text { control, } \\
B_{t} \text { is continuous, nondecreasing and } \\
B_{\iota}=\int_{0}^{t} 1_{\partial \Omega}\left(x_{\alpha}(s)\right) d \beta_{s} \quad \forall t \in \mathbb{R}
\end{array}\right.
$$

where $\alpha(t)$ is a measurable function from $[0, \infty)$ to $A$. The functions $u_{\lambda}(x)$ and $u(x, t)$ are given by

$$
\begin{array}{ll}
u_{\lambda}(x)=\inf _{\alpha} \int_{0}^{\infty} e^{-\lambda s} f\left(x_{\alpha}(s), \alpha(s)\right) d s & \lambda>0, x \in \bar{\Omega} \\
u(x, t)=\inf _{\alpha} \int_{0}^{t} f\left(x_{\alpha}(s), \alpha(s)\right) d s & x \in \bar{\Omega}, t \geq 0 .
\end{array}
$$

Remark that when $b(x, \alpha)=b(x), \forall x \in \bar{\Omega}$ the controlled system (9), (10) reduce to the ordinary differential equations. For time being, we restrict ourselves to the dynamical systems case i.e. $b(x, \alpha)=b(x), \forall x \in \bar{\Omega}$ and let $f(x, \alpha)=f(x), \forall x \in \bar{\Omega}$. In this case, the ergodicity is traditionally formulated in terms of measure theory. That is, denoting the evolution of the system as $T_{t}: x \rightarrow x(t), t \in \mathbb{R}$ from $\bar{\Omega}$ into $\bar{\Omega}$, taking an invariant measure $\mu_{0}$ (invariant under $T_{t}, t \geq 0$ ), the system is ergodic with respect to the measure $\mu_{0}$ when

$$
\lim _{T \rightarrow \infty} \frac{1}{T} \int_{0}^{T} f(x(t)) d t=\int_{\bar{\Omega}} f(x) d \mu_{0} \quad \mu_{0}-\text { a.e. } x \in \bar{\Omega}
$$

holds for any $f \in L^{1}\left(\bar{\Omega}, \mu_{0}\right)$, where $x(t)$ denotes the solution of (9) or (10) with $x(0)=x$ (see [1]). By recalling the following known relationship

$$
\lim _{\lambda \rightarrow 0} \lambda \int_{0}^{\infty} e^{-\lambda t} f(x(t)) d t=\lim _{T \rightarrow \infty} \frac{1}{T} \int_{0}^{T} f(x(t)) d t
$$

provided that at least one side is meaningful (a proof of this fact, named Abelian-Tauberian theorem can be found in [14]) we see that the convergence properties $\lim _{\lambda \rightarrow 0} \lambda u_{\lambda}(x)=d_{f}=\lim _{T \rightarrow \infty} \frac{1}{T} u(x, T)$ for $\forall x \in \bar{\Omega}$ relate closely to the ergodic theory. In fact, the system which has the above convergence properties is called uniquely ergodic ([6]). This 
is our reason to justify to use the usual terminology "ergodic" in calling the convergence problem of the terms $\lambda u_{\lambda}(x)$ and $\frac{1}{T} u(x, T)$ in the H.J.B. equations).

Now, we state our main result.

THEOREM 1. - Let $f(x, \alpha)$ in (1), (2) be in the form of $f(x, \alpha)=$ $f(x)+g(x, \alpha)$, where $f(x)$ is an arbitrary real-valued Lipschitz continuous function on $\bar{\Omega}$ and $g(x, \alpha)$ is a bounded continuous function in $\bar{\Omega} \times A$. If for any Lipschitz continuous function $f(x)$ there is a constant $d_{f}$ such that

$$
\begin{aligned}
\lim _{\lambda \rightarrow 0} \lambda u_{\lambda}(x) & =d_{f} \quad & \text { for all } x \in \bar{\Omega} \\
\left(\text { resp. } \lim _{T \rightarrow \infty} \frac{1}{T} u(x, T)\right. & =d_{f} & \text { for all } \quad x \in \bar{\Omega}
\end{aligned}
$$

then there is a subset $Z$ of $\bar{\Omega}$ which satisfies the following properties $(Z)$, $(P),(A)$.

(Z) $Z$ is non-empty and $z \in Z$ if and only if for any $y \in \bar{\Omega}$ and for any $\varepsilon>0$ there exist $T_{\varepsilon}>0$ and a control $\alpha_{\varepsilon}$ such that $\lim _{\varepsilon \rightarrow 0} T_{\varepsilon}=+\infty$, $\left|z-y_{\alpha_{\varepsilon}}\left(T_{\varepsilon}\right)\right|<\varepsilon$.

(P) $Z$ is closed, connected and positivity invariant i.e.

$\left(I_{+}\right) z_{\alpha}(t) \in Z \quad \forall x \in Z, \forall \alpha$ control,$\forall t \geq 0$.

(A) $Z$ has the following time averaged attracting property, i.e. for any open neighborhood $U$ of $Z$,

$$
\begin{aligned}
\lambda \int_{0}^{\infty} e^{-\lambda t} \chi_{U}\left(x_{\alpha}(t)\right) d t \rightarrow 1 & \\
& \text { as } \lambda \rightarrow 0, \text { uniformly in } \alpha, \forall x \in \bar{\Omega}, \\
(\text { resp. } & \frac{1}{T} \int_{0}^{T} \chi_{U}\left(x_{\alpha}(t)\right) d t \rightarrow 1 \\
& \text { as } T \rightarrow \infty, \text { uniformly in } \alpha, \forall x \in \bar{\Omega},)
\end{aligned}
$$

where $\chi_{U}(U \subset \bar{\Omega})$ denotes the characteristic function of the set $U$.

Concerning with Theorem 1, we make following remarks.

Remarks. - 1. From the property $(Z), Z$ is determined uniquely.

2. If $b(x, \alpha)=b(x) \quad \forall x \in \bar{\Omega}, \forall \alpha \in A$, then the following backward properties hold on $(Z)$.

$$
z(t) \in Z \quad \forall z \in Z, \forall t \in \mathbb{R}
$$

Vol. $14, n^{\circ} 4-1997$. 
For any $z_{1}, z_{2} \in Z$ and for any $\varepsilon>0$, there is a time $T_{\varepsilon}>0$ such that $\lim _{\varepsilon \rightarrow 0} T_{\varepsilon}=-\infty, \quad\left|z_{2}-z_{1}\left(T_{\varepsilon}\right)\right|<\varepsilon$.

We can explain the convergence property (15) (resp. (16)) by saying that there exists an invariant, connected subset $Z$ which is the attractor in the time averaged sense of $(A)$, that all the trajectories come as close as we wish to any point of $z \in Z$ infinitely many times. This qualitative property of the system matches very well with our intuitive understanding of ergodicity.

In the following section 2, we shall give some examples illustrating Theorem 1. After that, we state a more general result than theorem 1 when the convergence property (15) (resp. (16)) holds for a subset $\Omega_{0}$ of $\bar{\Omega}$ which does not depend on the choice of the continuous function $f(x, \alpha)$; we shall give also examples for this result. In section 3 , we shall prove theorems 1, 2 and the statements of the Remarks associated with these theorems. In section 4 , we shall give some general remarks concerning with the results in this paper.

In the following, we use the notations $\mathbb{R}, \mathbf{Z}, \mathbb{N}, \mathbb{R}^{+}$for the sets of real, integer, natural, positive real numbers respectively. The distance between two points $x, y \in \bar{\Omega}$ is given by $|x-y|$; the scalar product of $\mathbb{R}^{n} \times \mathbb{R}^{n}$ is denoted by $\langle\cdot, \cdot\rangle$. We use the letters $C\left(C_{1}, C_{2}, \ldots\right)$ for positive constants. For a Lipschitz continuous function $f(x), L_{f}$ denotes its Lipschitz constant. We shall write the solution of the O.D.Es. (9) or (10) as $x_{\alpha}(t), y_{\beta}(t)(t \in \mathbb{R})$ corresponding to the initial conditions $x_{\alpha}(0)=x, y_{\beta}(0)=y$ etc... When we consider a trajectory $x_{\alpha}(t)(t \in \mathbb{R})$, we call $\alpha$ a control for $x$. For $x \in \bar{\Omega}$, we denote by $U_{\varepsilon}(x)=\{y \in \bar{\Omega}|| x-y \mid<\varepsilon\}$. We shall sometimes write $H(x, p)=\sup _{\alpha \in A}\{-<b(x, \alpha), p>-f(x, \alpha)\}$ where the right-hand side appears in (1), (2).

From the Lipschitz continuity of $b(x, \alpha)$ in $x \in \bar{\Omega}$ and $\alpha \in A$, we have the following continuity of the controlled system.

$$
\left|x_{\alpha}(t)-y_{\alpha}(t)\right| \leq e^{\lambda_{0}|t|}|x-y| \quad \forall x, y \in \bar{\Omega}, \forall \alpha \text { control }, \forall t \in \mathbb{R}
$$

where $\lambda_{0}>0$ is a constant. In particular, in the periodic case $\lambda_{0}$ is given as follows (see P.L. Lions [10])

$$
\lambda_{0}=\sup _{\substack{x, x^{\prime} \in \bar{s} \\ \alpha \in A}}\left\{-<b(x, \alpha)-b\left(x^{\prime}, \alpha\right), x-x^{\prime}>\left|x-x^{\prime}\right|^{-2}\right\} .
$$

In the case of Neumann type B.C., the description of $\lambda_{0}$ is not so simple as in (22), in the case of state constraints B.C., we refer to the results of H.M. Soner [15]. 
To conclude this introduction, we shall give some references which relates with this subject. One can find the definition of the viscosity solution in [7], [10]. The existence and the uniqueness of the solutions of the H.J.B. equations (1), (2) with the above stated boundary conditions was obtained by P.L. Lions [11], G. Barles and P.L. Lions [2], H.M. Soner [15], I. Capuzzo-Dolcetta and P.L. Lions [4], P. Dupuis and H. Ishii [8]... For the treatment of the ergodic problem as the convergence problem in the H.J.B. equation, one can consult M. Robin [13] which contains many references, I. Capuzzo-Dolcetta and M.G. Garroni [3], I. Capuzzo-Dolcetta and J.L. Menaldi [5], P.L. Lions [11], P.L. Lions and B. Perthame [12] etc...

The author expresses her gratitude to Professeur Pierre-Louis Lions for his helpful advices and constant encouragements.

\section{EXAMPLES AND OTHER RESULT}

We give simple examples illustrating theorem 1 .

Example 1. - Let $\Omega$ be a bounded connected subregion on $\mathbb{R}^{n}$, and consider (1) (resp. (2)) with $H(p)=|p|$ with one of the boundary conditions of (3), (4) and (5) (resp. (3), (6) and (7)). By [11], we know that the system enjoys the convergence property (15) (resp. (16)) in theorem 1 with the uniform convergence. Therefore, by theorem 1 there exists a subset $Z \subset \bar{\Omega}$ which has the properties $(Z),(P)$ and $(A)$. In fact, by the form of $H(p)$ for all points $x, y \in \bar{\Omega}$ there exist a control $\alpha$ and a time $T>0$ such that $x_{\alpha}(T)=y$ and thus $Z=\bar{\Omega}$.

Example 2. - Let $\Omega$ be a bounded convex subregion of $\mathbb{R}^{n}$ containing the origin. Consider a positively definite symmetric linear operator $B$ on $\mathbb{R}^{n}$ and set $H(x, p)=\langle B x, p\rangle$. For the system given by (1) (resp. (2)) with this Hamiltonian and the boundary condition either one of (4) and (5) (resp. (6) and (7)). Then the system satisfies the convergence property (15) (resp. (16)) in Theorem 1 with uniform convergence. Therefore, by Theorem 1 there exists a subset $Z \subset \bar{\Omega}$ which has the propertics $(Z),(P)$ and $(A)$. In fact, in this system every point in $\bar{\Omega}$ is attracted to the origin and thus $Z=\{0\}$.

Example 3. - Let $\Omega$ be an open ball of radius 1 centered at the origin in $\mathbb{R}^{2}$. Consider (1) (resp. (2)) with $H(x, p)=\sup _{|\alpha| \leq 1}\left(\alpha p_{1}+x_{2} p_{2}\right)$ where $\alpha \in \mathbb{R}, p=\left(p_{1}, p_{2}\right)$ and either one of (4) and (5) (resp. (6) and (7)). Then the system satisfies the convergence property (15) (resp. (16)) in Vol. $14, n^{\circ}$ 4-1997. 
theorem 1 with the uniform convergence. Therefore, by theorem 1 , there exists a subset $Z \subset \bar{\Omega}$ which has the properties $(Z),(P)$ and $(A)$. In fact, in this system every point in $\bar{\Omega}$ is attracted to the $x_{2}$-axis and thus $Z=\left\{\left(x_{1}, x_{2}\right) \mid-1 \leq x_{1} \leq 1, x_{2}=0\right\}$.

Our second result is the following.

THEOREM 2. - Let $f(x, \alpha)$ in (1), (2) be in the form of $f(x, \alpha)-$ $f(x)+g(x, \alpha)$ where $f(x)$ is an arbitrary real-valued Lipschitz continuous function on $\bar{\Omega}$ and $g(x, \alpha)$ is a bounded continuous function in $\bar{\Omega} \times A$. Suppose that there exists a maximal subset $\Omega_{0}$ of $\bar{\Omega}$ such that for any Lipschitz continuous function $f(x)$ there exists a constant number $d_{f}$ such that

$$
\begin{aligned}
\lim _{\lambda \rightarrow 0} \lambda u_{\lambda}(x) & =d_{f} \quad \text { for all } x \in \Omega_{0} \\
\left(\text { resp. } \lim _{T \rightarrow \infty} \frac{1}{T} u(x, T)\right. & =d_{f} \quad \text { for all } x \in \Omega_{0}
\end{aligned}
$$

then there exists a subset $Z_{0}$ of $\bar{\Omega}$ which satisfies the following properties $\left(Z_{0}\right),\left(P_{0}\right)$ and $\left(A_{0}\right)$.

$\left(Z_{0}\right) Z_{0}$ is non-empty and $z \in Z_{0}$ if and only if for any $y \in \bar{\Omega}_{0}$ and for any $\varepsilon>0$ there exist $T_{\varepsilon}>0$ and a control $\alpha_{\varepsilon}$ such that $\lim _{\varepsilon \rightarrow 0} T_{\varepsilon}=+\infty$, $\left|z-y_{\alpha_{\varepsilon}}\left(T_{\varepsilon}\right)\right|<\varepsilon$.

$\left(P_{0}\right) Z_{0}$ is closed, positivity invariant i.e.

$\left(I_{+}\right) z_{\alpha}(t) \in Z_{0} \quad \forall z \in Z_{0}, \forall \alpha$ control, $\forall t \geq 0$.

$\left(A_{0}\right)$ For any open neighborhood $U$ of $Z_{0}$,

(24) (resp. $\varliminf_{T \rightarrow+\infty} \inf _{\alpha} \frac{1}{T} \int_{0}^{T} \chi_{U c}\left(x_{\alpha}(t)\right) d t=0 \quad \forall x \in \Omega_{0}$, )

where $\chi_{U}(U \subset \bar{\Omega})$ denotes the characteristic function of the set $U$.

Remarks. -4 . The properties $\left(Z_{0}\right),\left(P_{0}\right)$ and $\left(A_{0}\right)$ are weaker than that of $(Z),(P)$ and $(A)$ in theorem 1 , and here we do not know if $Z_{0} \subset \bar{\Omega}_{0}$. Moreover, we do not know if the following property holds on $Z_{0}$ :

for any points $z_{1}, z_{2} \in Z_{0}$ and for any $\varepsilon>0$

there exist $T_{\varepsilon}>0$ and a control $\alpha_{\varepsilon}$ such that

$\lim _{\varepsilon \rightarrow 0} T_{\varepsilon}=+\infty, \quad\left|z_{2}-z_{1_{\alpha_{\varepsilon}}}\left(T_{\varepsilon}\right)\right|<\varepsilon$. 
5. If $b(x, \alpha)=b(x), \forall x \in \bar{\Omega}, \forall \alpha \in A$, then the following stronger result holds: the subset $Z_{0} \subset \bar{\Omega}_{0}$ and in place of $\left(P_{0}\right),\left(A_{0}\right)$, we have $\left(P_{0}^{D}\right),\left(A_{0}^{D}\right)$ as follows.

$\left(P_{0}^{D}\right) Z_{0}$ is closed, invariant and connected.

$\left(A_{0}^{D}\right)$ For any neighborhood $U$ of $Z_{0}$,

$$
\begin{array}{rlrl}
\varlimsup_{\lambda \rightarrow 0} \lambda \int_{0}^{\infty} e^{-\lambda t} \chi_{U}(x(t)) d t & =1 & & \forall x \in \Omega_{0}, \\
\left(\operatorname{resp.} \varlimsup_{T \rightarrow+\infty} \frac{1}{T} \int_{0}^{T} \chi_{U}(x(l)) d t\right. & =1 & \forall x \in \Omega_{0} .
\end{array}
$$

We now give simple examples illustrating theorem 2 .

Example 4. - Let $\Omega$ be a bounded connected subregion of $\mathbb{R}^{n}$, and consider the equation (1) (resp. (2)) with $H(x, p)=a(x)|p|$ where $a(x)$ is a real-valued continuous function defined on $\bar{\Omega}$ satisfying that $a(x) \geq 0$, $a\left(x_{i}\right)=0,1 \leq i \leq N$ where $x_{i} \in \bar{\Omega}, a(x)>0$ in $\bar{\Omega} \backslash\left\{x_{1}, \ldots, x_{N}\right\}$, and wish one of the boundary condition given by (3), (4) or (5) (resp. (3), (6) or (7)). Then $\Omega_{0}=\bar{\Omega} \backslash\left\{x_{1}, \ldots, x_{N}\right\}, Z_{0}=\bar{\Omega}$.

Example 5. - Let $\Omega$ be a ball of radius 2 centcred at the origin in $\mathbb{R}^{n}$. Consider a negatively definite linear symmetric operator $B$ on $\mathbb{R}^{n}$ whose minimum eigenvalue is -1 , and set $H(x, p)=\{\langle B x, p\rangle+|p|\}$. Then for the equation (1) (resp. (2)) with this Hamiltonian and the boundary condition (4) (resp. (6)), $\Omega_{0}=\{x \in \Omega,|x|<1\}$ and $Z_{0}=\left\{x \in \mathbb{R}^{n},|x| \leq 1\right\}$.

Example 6. - Let $\Omega=(a, b)$ be an open interval in $\mathbb{R}$. For three numbers $c, d$, e such that $a<c<d<e<b$, put $g(x)=\int_{0}^{x}\left(x^{\prime}-c\right)\left(x^{\prime}-d\right)\left(x^{\prime}-e\right) d x^{\prime}$ and set $H(x, p)=<-\nabla g(x), p>$. Then there exist two disjointed subset $\Omega_{01}, \Omega_{02}$ in $\bar{\Omega}$ which have the convergence property (21), (22) in theorem 2 . For $\Omega_{01}$, the corresponding $Z_{01}=\{c\}$, for $\Omega_{02}$ the correspondig $Z_{02}=\{e\}$.

\section{PROOFS OF THEOREMS 1,2}

First, we shall prove theorem 1.

Proof of Theorem 1. - We shall prove the statements in the following three steps: (i) proof of $(Z)$; (ii) proof of $(P)$; (iii) proof of $(A)$. First of all, let us assume

$$
C_{2}<g(x, \alpha) \leq C_{1} \quad \forall x \in \bar{\Omega}, \forall \alpha \in A,
$$

where $C_{1} \geq C_{2}>0$ are fixed constants. 
(i) Proof of the property $(Z)$. - To prove this claim, we argue by contradiction: we assume that for any $x \in \bar{\Omega}$ there exist $\varepsilon(x)>0, T(x)>0$, a point $y(x) \in \bar{\Omega}$ such that

$$
y(x)_{\alpha}(t) \notin U_{\varepsilon(x)}(x) \quad \forall t>T(x), \forall \alpha \text { control. }
$$

Since $\bar{\Omega}$ is compact, there cxist a finite number of points $x_{1}, \ldots, x_{N} \in \bar{\Omega}$ such that $\bigcup_{k=1}^{N} U_{\frac{\xi\left(x_{k}\right)}{2}}\left(x_{k}\right) \supset \bar{\Omega}$. We shall denote by $\varepsilon_{k}=\frac{1}{2} \varepsilon\left(x_{k}\right), 1 \leq$ $k \leq N, \varepsilon=\min _{1 \leq k \leq N} \varepsilon_{k}, T_{k}=T\left(x_{k}\right) 1 \leq k \leq N, T_{0}=\max _{1 \leq k \leq N} T_{k}$, $y^{k}=y\left(x_{k}\right) 1 \leq k \leq N, U_{k}=U_{\varepsilon_{k}}\left(x_{k}\right), U_{k}^{\prime}=U_{\varepsilon_{k}+\varepsilon}\left(x_{k}\right)$. Then we have

$$
\bigcup_{k=1}^{N} U_{k} \supset \bar{\Omega}
$$

and for any $x_{k}(1 \leq k \leq N)$ there exists $y^{k} \in \bar{\Omega}(1 \leq k \leq N)$ such that

$$
y_{\alpha}^{k}(t) \notin U_{k}^{\prime} \quad \forall t>T_{0}, \forall \alpha \text { control, } 1 \leq k \leq N .
$$

In the following argument, we concentrate on the behavior of $y^{1}$. I et us denote $W=\left(\bigcup_{k=2}^{N} U_{k}\right) \cap\left(U_{1}^{\prime}\right)^{c} \cap \bar{\Omega}$. Since we can trivially assume that $\bigcup_{k=2}^{N} U_{k} \neq \emptyset$, the subset $W \subset \bar{\Omega}$ is non-empty. Moreover, since $W \cup U_{1}^{\prime} \supset \bar{\Omega}$, by (29) and from the boundary conditions of the system we have

$$
y_{\alpha}^{1}(t) \in W \quad \forall t>T_{0}, \forall \alpha \text { control. }
$$

Let $\left\{V_{\ell}^{\prime}\right\} 1 \leq \ell \leq M$ be a partition of $W$ composed of a finite number of open subsets of $W$ such that

$$
\begin{aligned}
& W=\bigcup_{k=1}^{M} V_{\ell}^{\prime}, \\
& V_{\ell}^{\prime} \cap V_{\ell^{\prime}}^{\prime}=\emptyset \quad \text { if } \ell \neq \ell^{\prime}, \\
& \partial V_{\ell}^{\prime} \subset\left(\bigcup_{k=2}^{N} \partial U_{k}\right) \cup \partial U_{1}^{\prime} \cup \partial \bar{\Omega} \quad \text { for } \quad 1 \leq \ell \leq M, \\
& V_{\ell}^{\prime} \cap\left\{\left(\bigcup_{k=2}^{N} \partial U_{k}\right) \cup \partial U_{1}^{\prime} \cup \partial \bar{\Omega}\right\}=\emptyset \quad \text { for } 1 \leq \ell \leq M,
\end{aligned}
$$

for example we can take $\left\{V_{\ell}^{\prime}\right\} 1 \leq \ell \leq M$ the finest family of open subsets of $W$ devided by the boundaries of $U_{k}(2 \leq k \leq N), U_{1}^{\prime}$ and $\bar{\Omega}$. 
Then, for any point $z_{0} \in W \backslash \bigcup_{\ell=1}^{M} V_{\ell}^{\prime}$, we shall choose arbitrary only one number $\ell_{0}\left(1 \leq \ell_{0} \leq M\right)$ such that $z_{0} \in \partial V_{\ell_{0}}^{\prime}$ and shall add $z_{0}$ to $V_{\ell_{0}}^{\prime}$. In this way, we can obtain a family of measurable subsets of $W$, say $\left\{V_{\ell}\right\} 1 \leq \ell \leq M$ where $V_{\ell}$ is the union of $V_{\ell}^{\prime}$ and some of the boundary points of $V_{\ell}^{\prime}$ satisfying

$$
W=\bigcup_{\ell=1}^{M} V_{\ell}, \quad V_{\ell} \cap V_{\ell^{\prime}}=\emptyset \quad \text { if } \quad \ell \neq \ell^{\prime} .
$$

For this family $\left\{V_{\ell}\right\} 1 \leq \ell \leq M$, we shall define the following values

$$
\begin{aligned}
L(\lambda, \alpha, \ell)= & \lambda \int_{0}^{\infty} e^{-\lambda t} \chi_{V_{\ell}}\left(y_{\alpha}^{1}(t)\right) d t \\
& \lambda>0, \alpha \text { control, } 1 \leq \ell \leq M
\end{aligned}
$$

$$
\begin{array}{r}
\text { (resp. } \tilde{L}(\lambda, \alpha, \ell)=\frac{1}{T} \int_{0}^{T} \chi_{V_{\ell}}\left(y_{\alpha}^{1}(t)\right) d t \\
T>0, \alpha \text { control, } 1 \leq \ell \leq M .)
\end{array}
$$

By (31), (32), (33)

$$
\lim _{\lambda \rightarrow 0} \sum_{\ell=1}^{M} L(\lambda, \alpha, \ell)=1 \quad \text { for all control } \alpha,
$$

$$
\text { (resp. } \left.\lim _{T \rightarrow \infty} \sum_{\ell=1}^{M} \tilde{L}(T, \alpha, \ell)=1 \quad \text { for all control } \alpha .\right)
$$

Thus, for an arbitrary fixed control $\alpha_{0}$, we can take a subsequence $\lambda^{\prime} \rightarrow 0$ (resp. $T^{\prime} \rightarrow \infty$ ) such that

$$
\begin{array}{ll}
(\text { resp. } & \lim _{T^{\prime} \rightarrow \infty} \tilde{L}\left(T^{\prime}, \alpha_{0}, \ell\right)=\tilde{L}\left(\alpha_{0}, \ell\right) \\
& \sum_{\ell=1}^{M} \tilde{L}\left(\alpha_{0}, \ell\right)=1 \quad \text { for } \quad 1 \leq \ell \leq M
\end{array}
$$

Vol. $14, \mathbf{n}^{\circ} 4-1997$. 
Hence, there is at least one $\ell_{0}, 1 \leq \ell_{0} \leq M$ such that

$$
\begin{gathered}
\frac{1}{M}<L\left(\alpha_{0}, \ell_{0}\right) \leq 1 . \\
\left(\text { resp. } \frac{1}{M}<\tilde{L}\left(\alpha_{0}, \ell_{0}\right) \leq 1 .\right)
\end{gathered}
$$

Choose a number $k_{0}, 2 \leq k_{0} \leq N$ such that $V_{\ell_{0}} \subset \bar{U}_{k_{0}}$ and take a Lipschitz continuous function $f(x)$ on $\bar{\Omega}$ such that

$$
\begin{aligned}
& 0 \leq f(x) \leq 2 M\left(C_{1}-C_{2}+1\right) \\
& f(x)=0 \text { in } \bar{U}_{k_{0}}, \quad=2 M\left(C_{1}-C_{2}+1\right) \text { in }\left(U_{k_{0}}^{\prime}\right)^{c} .
\end{aligned}
$$

Then by the convergence property (15) (resp. (16)) there must exist a number $d_{f}$ which satisfies the following two relationships at the same time. On one hand,

$$
\begin{aligned}
d_{f} & =\lim _{\lambda \rightarrow 0} \lambda u_{\lambda}\left(y^{1}\right) \\
& =\lim _{\lambda^{\prime} \rightarrow 0} \inf _{\alpha} \lambda^{\prime} \int_{0}^{\infty} e^{-\lambda^{\prime} t}\left(f\left(y_{\alpha}^{1}(t)\right)+g\left(y_{\alpha}^{1}(t), \alpha(t)\right)\right) d t \\
& \leq \varlimsup_{\lambda^{\prime} \rightarrow 0} \lambda^{\prime} \int_{0}^{\infty} e^{-\lambda^{\prime} t} f\left(y_{\alpha_{0}}^{1}(t)\right) d t+C_{1} \\
& =\varlimsup_{\lambda^{\prime} \rightarrow 0} \lambda^{\prime} \int_{0}^{\infty} e^{-\lambda^{\prime} t} f\left(y_{\alpha_{0}}^{1}(t)\right)\left(1-\chi_{U_{k_{0}}}\right)\left(y_{\alpha_{0}}^{1}(t)\right) d t+C_{1}
\end{aligned}
$$

and thus

$$
d_{f} \leq 2 M\left(C_{1}-C_{2}+1\right)\left(1-\frac{1}{M}\right)+C_{1}
$$

where we used (27), (36), (40). On the other hand, we have

$$
\begin{aligned}
d_{f} & =\lim _{\lambda \rightarrow 0} \lambda u_{\lambda}\left(y^{k_{0}}\right) \\
& =\lim _{\lambda \rightarrow 0} \inf _{\alpha}\left\{\lambda \int_{0}^{\infty} e^{-\lambda t}\left(f\left(y_{\alpha}^{k_{0}}(t)\right)+g\left(y_{\alpha}^{k_{0}}(t), \alpha(t)\right) d t\right\}\right. \\
& \geq \lim _{\lambda \rightarrow 0} \inf _{\alpha} \lambda \int_{0}^{\infty} e^{-\lambda t} f\left(y_{k_{0} \alpha}(t)\right) d t+C_{2}
\end{aligned}
$$

and thus

$$
d_{f} \geq 2 M\left(C_{1}-C_{2}+1\right)+C_{2}
$$


where we used (27), (29), (40). Thus, from (41), (42) we have

$$
2 M\left(C_{1}-C_{2}+1\right)+C_{2} \leq 2 M\left(C_{1}-C_{2}+1\right)\left(1-\frac{1}{M}\right)+C_{1}
$$

which is clearly a contradiction with (27).

Similarly, in the finite horizon case we have

$$
\begin{aligned}
d_{f} & =\lim _{T \rightarrow \infty} \frac{1}{T} u\left(y^{1}, T\right) \\
& =\lim _{T^{\prime} \rightarrow \infty} \inf \left\{\frac{1}{T^{\prime}} \int_{0}^{T^{\prime}} f\left(y_{\alpha}^{1}(t)\right)+g\left(y_{\alpha}^{1}(t), \alpha(t)\right) d t\right\} \\
& \leq \varlimsup_{T^{\prime} \rightarrow \infty} \frac{1}{T^{\prime}} \int_{0}^{T^{\prime}} f\left(y_{\alpha_{0}}^{1}(t)\right) d t+C_{1} \\
& =\varlimsup_{T^{\prime} \rightarrow \infty} \frac{1}{T^{\prime}} \int_{0}^{T^{\prime}} f\left(y_{\alpha_{0}}^{1}(t)\right)\left(1-\chi_{U_{k_{0}}}\right)\left(y_{\alpha_{0}}^{1}(t)\right) d t+C_{1}
\end{aligned}
$$

and thus

$$
d_{f} \leq 2 M\left(C_{1}-C_{2}+1\right)\left(1-\frac{1}{M}\right)+C_{1}
$$

where we used (29), (39), (42). On the other hand,

$$
\begin{aligned}
d_{f} & =\lim _{T \rightarrow \infty} \frac{1}{T} u\left(y^{k_{0}}, T\right) \\
& =\lim _{T \rightarrow \infty} \inf _{\alpha}\left\{\frac{1}{T} \int_{0}^{T} f\left(y_{\alpha}^{k_{0}}(t)\right)+g\left(y_{\alpha}^{k_{0}}(t), \alpha(t)\right) d t\right\} \\
& \geq \lim _{T \rightarrow \infty} \inf _{\alpha} \frac{1}{T} \int_{0}^{T} f\left(y_{\alpha}^{k_{0}}(t)\right) d t+C_{2}
\end{aligned}
$$

and thus

$$
d_{f} \geq 2 M\left(C_{1}-C_{2}+1\right)+C_{2}
$$

where we used (27), (29), (40). And as in the argument for the infinite horizon case we reach a contradiction.

(ii) Proof of the property $(P)$. - First, we shall prove the closedness. Let $z_{n} \in Z$ be a sequence such that $\lim _{n \rightarrow \infty} z_{n}=z_{\infty} \in \bar{\Omega}$. We are to show that $z_{\infty} \in Z$. Let $x \in \bar{\Omega}$ be arbitrary and $\varepsilon>0$ be arbitrary. We take a number $n_{0} \in \mathbf{N}$ large enough so that $\left|z_{n_{0}}-z_{\infty}\right|<\frac{\varepsilon}{2}$ holds. By the property $(Z)$, since $z_{n_{0}} \in Z$ there exist $T>0$ and a control $\alpha$ for $x$ such 
that $x_{\alpha}(T) \in U_{\frac{\varepsilon}{2}}\left(z_{n_{0}}\right)$. Clearly, $x_{\alpha}(T) \in U_{\varepsilon}\left(z_{\infty}\right)$ and since $x \in \bar{\Omega}, \varepsilon>0$ are arbitrary we have proved that $z_{\infty} \in Z$.

Next, we shall prove the positive invariance $\left(I_{+}\right)$of $Z$. Let $z \in Z, \alpha$ control for $z, T>0$. We want to show that $z_{\alpha}(T) \in Z$. Let $x \in \bar{\Omega}$, $\varepsilon>0$ be arbitrary and set $\varepsilon^{\prime}=\exp \left(-\lambda_{0} T\right) \varepsilon$ where $\lambda_{0}>0$ is the constant appearing in (21). For this $\varepsilon^{\prime}$, we derive from $(Z)$ that there exist $T_{1}>0$ and a control $\beta$ for $x$ such that

$$
T_{1}+T>0, \quad x_{\beta}\left(T_{1}\right) \in U_{\varepsilon^{\prime}}(z)
$$

We denote $x_{1}=x_{\beta}\left(T_{1}\right)$. By (19),

$$
\left|x_{1 \alpha}(T)-z_{\alpha}(T)\right| \leq e^{\lambda_{0} T}\left|x_{1}-z\right|=\varepsilon .
$$

Now, denoting the control $\bar{\alpha}(t)=\beta(t), 0 \leq t \leq T_{1} ;=\alpha\left(t-T_{1}\right)$, $T_{1} \leq t \leq T_{1}+T$, from (48) we have $x_{\bar{\alpha}}\left(T_{1}+T\right) \in U_{\varepsilon}\left(z_{\alpha}(T)\right)$. Since $x \in \bar{\Omega}, \varepsilon>0$ are arbitrary we have proved $z_{\alpha}(T) \in Z$.

Finally, we shall prove that $Z$ is connected. Assume that there are two open subsets of $\bar{\Omega}: U_{1}, U_{2}$ such that $U_{1} \cap Z \neq \emptyset, U_{2} \cap Z \neq \emptyset, U_{1} \cap U_{2}=\emptyset$, $Z \subset U_{1} \cup U_{2}$. Let $z_{1} \in Z \cap U_{1}, z_{2} \in Z \cup U_{2}$ be arbitrary. Choose $\varepsilon>0$ small enough so that $U_{\varepsilon}\left(z_{2}\right) \in U_{2}$. Then, by the property $(Z)$ there exist $T_{1}>0$, a control $\alpha_{1}$ for $z_{1}$ such that $z_{1 \alpha_{1}}(t) \in U_{\varepsilon}\left(z_{2}\right)$. However, this is impossible because the set $\left\{z_{1} \alpha_{1} \mid 0 \leq t \leq T_{1}\right\}$ is a connected set which must be contained in $U_{1}$. Therefore, the above assumption leads to a contradiction and $Z$ is connected.

(iii) Proof of the property $(A)$. - We assume that the property $(A)$ does not hold and we shall look for a contradiction. We use the facts that $Z$ is closed and invariant which we have proved in (ii). If (17) (resp. (18)) does not hold, there are open subsets $U_{1}, U_{2} \subset \bar{\Omega}$, a point $\bar{x} \in \bar{\Omega}$, a constant $C_{3}>0$ such that

$$
\begin{gathered}
Z \subset U_{1} \subseteq U_{2}, \\
\varlimsup_{\lambda \rightarrow 0} \lambda \sup _{\alpha} \int_{0}^{\infty} e^{-\lambda t} \chi_{U_{2}^{c}}\left(\bar{x}_{\alpha}(t)\right) d t=C_{3}>0 . \\
\left(\text { resp. } Z \subset U_{1} \subseteq U_{2},\right. \\
\left.\varlimsup_{T \rightarrow \infty} \frac{1}{T} \sup _{\alpha} \int_{0}^{T} \chi_{U_{2}^{c}}\left(\bar{x}_{\alpha}(t)\right) d t=C_{3}>0 .\right)
\end{gathered}
$$


Take a Lipschitz continuous function $f(x)$ on $\bar{\Omega}$ such that

$$
\begin{aligned}
& 0 \leq f(x) \leq \frac{1}{C_{3}}\left(C_{1}-C_{2}+1\right) \quad \text { in } \bar{\Omega} \\
& f(x)=\frac{1}{C_{3}}\left(C_{1}-C_{2}+1\right) \quad \text { in } U_{1},=0 \quad \text { in } U_{2}^{c} .
\end{aligned}
$$

Then by (27), (49) and by the invariance of $Z$, for any $z \in Z$

$$
\begin{aligned}
\lim _{\lambda \rightarrow 0} \lambda u_{\lambda}(z) & =\lim _{\lambda \rightarrow 0} \inf _{\alpha}\left\{\lambda \int_{0}^{\infty} e^{-\lambda t}\left(f\left(z_{\alpha}(t)\right)+g\left(z_{\alpha}(t), \alpha(t)\right) d t\right\}\right. \\
& \geq \frac{1}{C_{3}}\left(C_{1}-C_{2}+1\right)+C_{2},
\end{aligned}
$$

and by (27), (47), (49),

$$
\begin{aligned}
\lim _{\lambda \rightarrow 0} \lambda u_{\lambda}(\bar{x}) & =\lim _{\lambda \rightarrow 0} \inf _{\alpha}\left\{\lambda \int_{0}^{\infty} e^{-\lambda t}\left(f\left(\bar{x}_{\alpha}(t)\right)+g\left(\bar{x}_{\alpha}(t), \alpha(t)\right)\right) d t\right\} \\
& \leq \varlimsup_{\lambda \rightarrow 0} \inf _{\alpha} \lambda \int_{0}^{\infty} e^{-\lambda t} f\left(\bar{x}_{\alpha}(t)\right) d t+C_{1} \\
& =\varlimsup_{\lambda \rightarrow 0} \inf _{\alpha}\left\{\lambda \int_{0}^{\infty} e^{-\lambda t} f\left(\bar{x}_{\alpha}(t)\right)\left(1-\chi_{U_{2}}\right)\left(\bar{x}_{\alpha}(t)\right) d t\right\}+C_{1}
\end{aligned}
$$

and thus

$$
\lim _{\lambda \rightarrow 0} \lambda u_{\lambda}(\bar{x}) \leq \frac{1}{C_{3}}\left(C_{1}-C_{2}+1\right)\left(1-C_{3}\right)+C_{1} .
$$

We thus reach a contradiction and we have proved the property $(A)$, which completes the proof of Theorem 1.

Next, we shall prove the assertion of Remark 2.

Proof of Remark 2. - To check the invariant property (I), by $\left(I_{+}\right)$it is enough to show that $z(t) \in Z$ for all $z \in Z$, all $t<0$. So, let $z \in Z, T<0$ be arbitrary. Let $x \in \bar{\Omega}, \varepsilon>0$ be also arbitrary and let $\varepsilon^{\prime}=\exp \left(\lambda_{0} T\right) \varepsilon$ where $\lambda_{0}>0$ is the constant appearing in (19). By the property $(Z)$, there is a time $T_{1}>0$ large enough such that

$$
T_{1}+T>0, \quad x\left(T_{1}\right) \in U_{\varepsilon^{\prime}}(z)
$$

Denoting $x_{1}=x\left(T_{1}\right)$, by (21) we have

$$
\left|x_{1}(T)-z(T)\right| \leq e^{\lambda_{0}|T|}\left|x_{1}-z\right|=\varepsilon,
$$

that is we have $\left|x\left(T_{1}+T\right)-z(T)\right| \leq \varepsilon$. Since $x \in \bar{\Omega}, \varepsilon>0$ are arbitrary and we can take $T_{1}>0$ as large as necessary, we have proved that $z(T) \in Z$. Vol. 14, $\mathrm{n}^{\circ} 4-1997$. 
Next, we shall prove the property $\left(Z^{-}\right)$. Let $z_{1}, z_{2} \in Z, \varepsilon>0$ be arbitrary chosen. By $(I),\left\{z_{1}(t) \mid t<0\right\}<Z$ and thus by the compactness of $Z$ there is a sequence $\left\{t_{n}\right\}_{n \in \mathbf{N}}, \lim _{n \rightarrow \infty}, t_{n}=-\infty$ and a point $z_{-\infty} \in Z$ such that $\lim _{n \rightarrow \infty} z_{1}\left(t_{n}\right)=z_{-\infty}$. By the property $(Z)$, there is a time $T>0$ such that

$$
z_{-\infty}(T) \in U_{\frac{c}{2}}\left(z_{2}\right) .
$$

Let $\varepsilon^{\prime}=\frac{1}{2} \exp \left(-\lambda_{0} T\right) \varepsilon$, where $\lambda_{0}>0$ is a constant appearling in (19). Let us take a number $n^{\prime} \in \mathbf{N}$ large enough so that $z_{1}\left(t_{n}^{\prime}\right) \in U_{\varepsilon^{\prime}}\left(z_{-\infty}\right)$. Then by (19), denoting $z_{n}^{\prime}=z_{1}\left(t_{n}^{\prime}\right)$ we have

$$
\left|z_{n}^{\prime}(T)-z_{-\infty}(T)\right| \leq e^{\lambda_{0} T}\left|z_{n}^{\prime}-z_{-\infty}\right|=\frac{\varepsilon}{2} .
$$

By (53), (54), we get $z_{1}\left(t_{n}^{\prime}+T\right) \in U_{\varepsilon}\left(z_{2}\right)$ and since $\varepsilon>0$ is arbitrary and $t_{n}^{\prime}<0$ can be taken as small as we wish independtly on the choise of $T$, we have proved the property $\left(Z^{-}\right)$. Therefore, the assertion in Remark 1 was proved.

Now, we shall prove Theorem 2.

Proof of Theorem 2. - The former two properties $\left(Z_{0}\right),\left(P_{0}\right)$ can be proved as in the proof of Theorem 1. In fact, we only change $\bar{\Omega}$ in the former proof to $\Omega_{0}$, so we do not repeat them. Here, we only prove the property $\left(A_{0}\right)$. Let $C_{1} \geq C_{2}>0$ be the constants such that

$$
C_{2} \leq g(x, \alpha) \leq C_{1} \quad \forall x \in \bar{\Omega}, \forall \alpha \in A .
$$

The difference with Theorem 1 comes from the fact that we do not know if $Z_{0} \subseteq \Omega_{0}$ (and in general it is not true !). Let us assume that $\left(A_{0}\right)$ does not hold, and we shall look for a contradiction. We use the fact that $Z_{0}$ is closed (obtained in $\left(P_{0}\right)$ ). We assume that there exist three open subsets $U_{1}, U_{2}, U_{3} \subset \bar{\Omega}$, a point $\bar{x} \in \Omega_{0}$ and a number $C_{3}>0$ such that

$$
\begin{gathered}
Z \subset U_{1} \subseteq U_{2} \subseteq U_{3}, \quad U_{1} \cap U_{3}^{c}=\emptyset \\
\varliminf_{\lambda \rightarrow 0} \inf _{\alpha} \lambda \int_{0}^{\infty} e^{-\lambda t} \chi_{U_{3}^{\prime}}\left(\bar{x}_{\alpha}(t)\right) d t=C_{3}>0 \\
\left(\text { resp. } \varliminf_{\lambda \rightarrow 0} \inf _{\alpha} \frac{1}{T} \int_{0}^{\infty} \chi_{U_{3}^{\prime}}\left(\bar{x}_{\alpha}(t)\right) d t=C_{3}>0 .\right)
\end{gathered}
$$


Take a Lipschitz continuous function $f(x)$ defined on $\bar{\Omega}$ such that

$$
\begin{aligned}
& 0 \leq f(x) \leq C_{1} / C_{3}, \quad \operatorname{supp} f(x) \subset U_{2}, \\
& f(x)=0 \quad \text { in } U_{1},=\frac{C_{1}}{C_{3}} \text { in } U_{3}^{c} .
\end{aligned}
$$

By the convergence property (21) (resp. (22)), we have by (55), (56) (resp. (57)), (61),

$$
\begin{aligned}
\lim _{\lambda \rightarrow 0} \lambda u_{\lambda}(x) & =\lim _{\lambda \rightarrow 0} \inf _{\alpha} \lambda \int_{0}^{\infty} e^{-\lambda t} f\left(\bar{x}_{\alpha}(t)\right)+g\left(\bar{x}_{\alpha}(t), \alpha(t)\right) d t \\
& \geq \varliminf_{\lambda \rightarrow 0} \inf _{\alpha} \lambda \int_{0}^{\infty} e^{-\lambda t} f\left(\bar{x}_{\alpha}(t)\right) d t+C_{2} \\
& \geq \varliminf_{\lambda \rightarrow 0} \inf _{\alpha} \lambda \int_{0}^{\infty} e^{-\lambda t} \times \frac{C_{1}}{C_{3}} \chi_{U_{3}^{c}}\left(\bar{x}_{\alpha}(t)\right) d t+C_{2}
\end{aligned}
$$

and thus

$$
\lim _{\lambda \rightarrow 0} \lambda u_{\lambda}(x) \geq C_{1}+C_{2} \quad \text { for all } x \in \Omega_{0} .
$$

Similarly, for the finite horizon case

$$
\begin{aligned}
\lim _{T \rightarrow \infty} \frac{1}{T} u(x, T) & =\lim _{T \rightarrow \infty} \inf _{\alpha} \frac{1}{T} \int_{0}^{T} f\left(\bar{x}_{\alpha}(t)\right)+g\left(\bar{x}_{\alpha}(t), \alpha(t)\right) d t \\
& \geq \underset{T \rightarrow \infty}{\lim _{\alpha}} \inf _{\alpha} \frac{1}{T} \int_{0}^{T} f\left(\bar{x}_{\alpha}(t)\right) d t+C_{2},
\end{aligned}
$$

and thus

$$
\lim _{T \rightarrow \infty} \frac{1}{T} u(x, T) \geq C_{1}+C_{2} \quad \text { for all } \quad x \in \Omega_{0} .
$$

On the other hand, for any $\lambda>0$ (resp. $T>0$ ) by (58)

$$
\begin{aligned}
\inf _{\alpha} \lambda & \int_{0}^{\infty} e^{-\lambda t} \times \frac{C_{1}}{C_{3}} \chi_{U_{2}^{c}}\left(x_{\alpha}(t)\right) d t \\
& \geq \inf _{\alpha} \lambda \int_{0}^{\infty} e^{-\lambda t} f\left(x_{\alpha}(t)\right) d t \quad \forall x \in \Omega_{0},
\end{aligned}
$$

(62) (resp. $\inf _{\alpha} \frac{1}{T} \int_{0}^{T} \frac{C_{1}}{C_{3}} \chi_{U_{2}^{c}}\left(x_{\alpha}(t)\right) d t$

$$
\left.\geq \inf _{\alpha} \frac{1}{T} \int_{0}^{T} f\left(x_{\alpha}(t)\right) d t \quad \forall x \in \Omega_{0} .\right)
$$


And by (59), (61) (resp. (60), (62)) we have

$$
\begin{aligned}
& \underset{\lim _{\lambda \rightarrow 0}}{ } \inf _{\alpha} \lambda \int_{0}^{\infty} e^{-\lambda t} \times \frac{C_{1}}{C_{3}} \chi_{U_{2}}\left(x_{\alpha}(t)\right) d t \\
& \quad \geq \varliminf_{\lambda \rightarrow 0} \inf _{\alpha} \lambda \int_{0}^{\infty} e^{-\lambda t} f\left(x_{\alpha}(t)\right) d t \geq C_{2} \quad \forall x \in \Omega_{0} .
\end{aligned}
$$

$$
\text { (resp. } \begin{aligned}
\underline{\lim } \inf _{T \rightarrow \infty} & \frac{1}{T} \int_{0}^{T} \frac{C_{1}}{C_{3}} \chi_{U_{2}^{c}}\left(x_{\alpha}(t)\right) d t \\
& \left.\geq \underset{T \rightarrow \infty}{\lim _{\alpha}} \inf _{\alpha} \frac{1}{T} \int_{0}^{T} f\left(x_{\alpha}(t)\right) d t \geq C_{2} \quad \forall x \in \Omega_{0} .\right)
\end{aligned}
$$

In fact, for any $x \in \Omega_{0}$ let us take a subsequence $\lambda^{\prime} \rightarrow 0\left(\right.$ resp. $T^{\prime} \rightarrow \infty$ ) such that

$$
\begin{gathered}
\varliminf_{\lambda \rightarrow 0} \inf _{\alpha} \lambda \int_{0}^{\infty} e^{-\lambda t} f\left(x_{\alpha}(t)\right) d t=\lim _{\lambda^{\prime} \rightarrow 0} \inf _{\alpha} \lambda^{\prime} \int_{0}^{\infty} e^{-\lambda^{\prime} t} f\left(x_{\alpha}(t)\right) d t, \\
\text { (resp. } \left.\underline{\lim }_{T \rightarrow \infty} \inf _{\alpha} \frac{1}{T} \int_{0}^{T} f\left(x_{\alpha}(t)\right) d t=\lim _{T^{\prime} \rightarrow \infty} \inf _{\alpha} \frac{1}{T^{\prime}} \int_{0}^{T^{\prime}} f\left(x_{\alpha}(t)\right) d t,\right)
\end{gathered}
$$

and then by using this sequence $\lambda^{\prime} \rightarrow 0$ (resp. $T^{\prime} \rightarrow \infty$ ) we deduce from (59) (resp. (60))

$$
\begin{aligned}
C_{1}+C_{2} & \leq \lim _{\lambda \rightarrow 0} \lambda u_{\lambda}(x)=\lim _{\lambda^{\prime} \rightarrow 0} \lambda^{\prime} u_{\lambda^{\prime}}(x) \\
& =\lim _{\lambda^{\prime} \rightarrow 0} \inf _{\alpha} \lambda^{\prime} \int_{0}^{\infty} e^{-\lambda^{\prime} t}\left(f\left(x_{\alpha}(t)\right)+g\left(x_{\alpha}(t), \alpha(t)\right)\right) d t \\
& \leq \lim _{\lambda^{\prime} \rightarrow 0} \inf _{\alpha} \lambda^{\prime} \int_{0}^{\infty} e^{-\lambda^{\prime} t} f\left(x_{\alpha}(t)\right) d t+C_{1} \quad \text { for any } x \in \Omega_{0},
\end{aligned}
$$

(resp. $\quad C_{1}+C_{2} \leq \lim _{T \rightarrow \infty} \frac{1}{T} u(x, T)=\lim _{T^{\prime} \rightarrow \infty} \frac{1}{T^{\prime}} u\left(x, T^{\prime}\right)$

$$
\begin{aligned}
& =\lim _{T^{\prime} \rightarrow \infty} \inf _{\alpha} \frac{1}{T^{\prime}} \int_{0}^{T^{\prime}} f\left(x_{\alpha}(t)\right)+g\left(x_{\alpha}(t), \alpha(t)\right) d t \\
& \left.\leq \lim _{T^{\prime} \rightarrow \infty} \inf _{\alpha} \frac{1}{T^{\prime}} \int_{0}^{T^{\prime}} f\left(x_{\alpha}(t)\right) d t+C_{1} \quad \text { for any } x \in \Omega_{0}\right)
\end{aligned}
$$

and we have (63) (resp. (64)).

At this stage, from (63) (resp. (64), we can derive $U_{2}^{c} \cap Z \neq \emptyset$ which is apparently a contradiction. In other words, we can show that there exists a point $\tilde{z} \in U_{2}^{c}$ such that for any point $x \in \Omega_{0}$ and for any $\varepsilon>0$ there exist $T_{\varepsilon}>0$ and a control $\alpha_{\varepsilon}$ for $x$ such that $x_{\alpha_{\varepsilon}}\left(T_{\varepsilon}\right) \in U_{\varepsilon}(\tilde{z})$. For 
this, we follow the argument used in Theorem 1 to prove the property $(Z)$. That is, we assume the contrary: $\left\{z \in U_{2}^{c} \mid\right.$ for any point $x \in \Omega_{0}$ and for any $\varepsilon>0$, there exist $T_{\varepsilon}>0$ and a control $\alpha_{\varepsilon}$ such that $\left.x_{\alpha_{\varepsilon}}\left(T_{c}\right) \in U_{\varepsilon}(z)\right\}=\emptyset$, and we shall look for a contradiction. As in the proof of Theorem 1, the above assumption leads the existence of the finite points $x_{1}, \ldots, x_{N} \in U_{2}^{c}, y^{K} \in \Omega_{0}(1 \leq k \leq N)$, a finite number of real numbers $\varepsilon_{k}>0(1 \leq k \leq N), \varepsilon=\min _{1 \leq k \leq N} \varepsilon_{k}, T_{k}>0(1 \leq k \leq N)$, $T_{0}=\max _{1 \leq k \leq N} T_{k}$, a finite number of open sets $\tilde{U}_{k}=U_{\varepsilon_{k}}\left(x_{k}\right) \cap U_{2}^{c}$ and $\tilde{U}_{k}^{\prime}=U_{\varepsilon_{k}+\varepsilon}\left(x_{k}\right) \cap U_{2}^{c}(1 \leq k \leq N)$ such that

$$
\bigcup_{k=1}^{N} \tilde{U}_{k}=U_{2}^{c}
$$

and for any $x_{k}(1 \leq k \leq N)$

$$
y_{\alpha}^{k}(t) \notin \tilde{U}_{k}^{\prime} \quad \forall t>T_{0}, \forall \alpha \text { control, } 1 \leq k \leq N .
$$

We also denote $W=\left(\bigcup_{k=2}^{N} \tilde{U}_{k}\right) \cap\left(\tilde{U}_{1}^{\prime}\right)^{c} \cap\left(U_{2}^{c}\right)$. Since we can trivially assume that $\bigcup_{k=2}^{N} \tilde{U}_{k} \neq \emptyset$, the subset $W \subset U_{2}^{c}$ is non-empty. Moreover, since

$$
W \cap \tilde{U}_{1}^{\prime}=U_{2}^{c}
$$

by (63) (resp. (64)) and (66)

$$
\varliminf_{\lambda \rightarrow 0} \inf _{\alpha} \lambda \int_{0}^{\infty} e^{-\lambda \iota} \chi_{W}\left(y_{\alpha}^{1}(t)\right) d t \geq \frac{C_{2} C_{3}}{C_{1}} .
$$

$$
\text { (resp. } \left.\varliminf_{T \rightarrow \infty} \inf _{\alpha} \frac{1}{T} \int_{0}^{T} \chi_{W}\left(y_{\alpha}^{1}(t)\right) d t \geq \frac{C_{2} C_{3}}{C_{1}} .\right)
$$

Now, as in the proof of Theorem 1, we take a family of subsets of $W$, say $\left\{V_{\ell}\right\}(1 \leq \ell \leq M)$ satisfying

$$
\begin{aligned}
& W=\bigcup_{\ell=1}^{M} V_{\ell}, \quad V_{\ell} \cap V_{\ell^{\prime}}=\emptyset \quad \text { if } \quad \ell \neq \ell^{\prime}, \\
& \partial V_{\ell} \subset\left(\bigcup_{k=2}^{N} \partial \tilde{U}_{k}\right) \cup \partial \tilde{U}_{1}^{\prime} \cup \partial\left(U_{2}^{c}\right) \quad 1 \leq \ell \leq M, \\
& \bigcup_{\ell=1}^{M} \partial V_{\ell}=\left(\bigcup_{k=2}^{N} \partial \tilde{U}_{k}\right) \cup \partial \tilde{U}_{1}^{\prime} \cup \partial\left(U_{2}^{c}\right) .
\end{aligned}
$$


For this family $\left\{V_{\ell}\right\}(1 \leq \ell \leq M)$, we shall define the following values

$$
\begin{gathered}
L(\lambda, \alpha, \ell)=\lambda \int_{0}^{\infty} e^{-\lambda t} \chi_{V_{i}}\left(y_{\alpha}^{1}(t)\right) d t \\
\lambda>0, \alpha \text { control, } 1 \leq \ell \leq M
\end{gathered}
$$

$$
\begin{aligned}
(\text { resp. } \tilde{L}(T, \alpha, \ell) & =\frac{1}{T} \int_{0}^{T} \chi_{V_{\ell}}\left(y_{\alpha}^{\prime}(t)\right) d t \\
T & >0, \alpha \text { control, } 1 \leq \ell \leq M .)
\end{aligned}
$$

By (67), (69), (70) (resp. (68), (69), (70)) for any fixed control $\alpha_{0}$ we have

$$
\varliminf_{\lambda \rightarrow 0} \sum_{\ell=1}^{M} L\left(\lambda, \alpha_{0}, \ell\right) \geq \frac{C_{2} C_{3}}{C_{1}}
$$

$$
\left(\text { resp. } \lim _{T \rightarrow \infty} \sum_{\ell=1}^{M} \tilde{L}\left(T, \alpha_{0}, \ell\right) \geq \frac{C_{2} C_{3}}{C_{1}},\right)
$$

and we can take a subsequence $\lambda^{\prime} \rightarrow 0$ (resp. $T^{\prime} \rightarrow \infty$ ) such that

$$
\begin{aligned}
& \lim _{\lambda^{\prime} \rightarrow 0} L\left(\lambda^{\prime}, \alpha_{0}, \ell\right)=\exists L\left(\alpha_{0}, \ell\right) \quad 1 \leq \ell \leq M, \\
& \sum_{\ell=1}^{M} L\left(\alpha_{0}, \ell\right)=\frac{C_{2} C_{3}}{C_{1}} .
\end{aligned}
$$

$$
\begin{array}{cl}
\text { (resp. } & \lim _{T^{\prime} \rightarrow \infty} \tilde{L}\left(T^{\prime}, \alpha_{0}, \ell\right)=\exists \tilde{L}\left(\alpha_{0}, \ell\right) \quad 1 \leq \ell \leq M, \\
& \left.\sum_{\ell=1}^{M} \tilde{L}\left(\alpha_{0}, \ell\right)=\frac{C_{2} C_{3}}{C_{1}}\right)
\end{array}
$$

Therefore, there is at least one $\ell_{0}\left(1 \leq \ell_{0} \leq M\right)$ such that

$$
\begin{gathered}
L\left(\alpha_{0}, \ell_{0}\right)>\frac{C_{2} C_{3}}{M C_{1}}, \\
\left(\text { resp. } \tilde{L}\left(\alpha_{0}, \ell_{0}\right)>\frac{C_{2} C_{3}}{M C_{1}},\right)
\end{gathered}
$$


and we choose a number $k_{0}\left(2 \leq k_{0} \leq N\right)$ such that $V_{\ell_{0}} \subset \overline{\tilde{U}}_{k_{0}}$. Now, we take a Lipschitz continuous function $f(x)$ on $\bar{\Omega}$ such that

$$
\begin{aligned}
f(x) \quad=0 & \text { in } \tilde{U}_{k_{0}} \\
=\frac{M C_{1}}{C_{2} C_{3}}\left(C_{1}-C_{2}+1\right) & \text { in }\left(\tilde{U}_{k_{0}}^{\prime}\right)^{\circ} \\
0 \leq f(x) \leq \frac{M C_{1}}{C_{2} C_{3}}\left(C_{1}-C_{2}+1\right) & \text { in } \bar{\Omega}
\end{aligned}
$$

Then, by the convergence property (21) (resp. (22)) there must exist a real number $d_{f}$ which satisfies the following two relationships at the same time. On one hand,

$$
\begin{aligned}
d_{f} & =\lim _{\lambda \rightarrow 0} \lambda u_{\lambda}\left(y^{1}\right) \\
& =\lim _{\lambda^{\prime} \rightarrow 0} \lambda^{\prime} \inf _{\alpha} \int_{0}^{\infty} e^{-\lambda^{\prime} t}\left(f\left(y_{\alpha}^{1}(t)\right)+g\left(y_{\alpha}^{1}(t), \alpha(t)\right) d t\right. \\
& \leq \varlimsup_{\lambda^{\prime} \rightarrow 0} \lambda^{\prime} e^{-\lambda^{\prime} t} f\left(y_{\alpha_{0}}^{1}(t)\right) d t+C_{1} \\
& =\varlimsup_{\lambda^{\prime} \rightarrow 0} \lambda^{\prime} \int_{0}^{\infty} e^{-\lambda^{\prime} t} f\left(y_{\alpha_{0}}^{1}(t)\right)\left(1-\chi_{\tilde{U}_{k_{0}}}\right)\left(y_{\alpha_{0}}^{1}(t)\right) d t+C_{1}
\end{aligned}
$$

and thus

$$
d_{f} \leq \frac{M C_{1}}{C_{2} C_{3}}\left(C_{1}-C_{2}+1\right)\left(1-\frac{C_{2} C_{3}}{M C_{1}}\right)+C_{1}
$$

where we used $(55),(70),(74),(76)$ and (78). On the other hand,

$$
\begin{aligned}
d_{f} & =\lim _{\lambda \rightarrow 0} \lambda u_{\lambda}\left(y^{k_{0}}\right) \\
& =\lim _{\lambda \rightarrow 0} \inf _{\alpha}\left\{\lambda \int_{0}^{\infty} e^{-\lambda t} f\left(y_{\alpha}^{k_{0}}(t)\right)+g\left(y_{\alpha}^{k_{0}}(t), \alpha(t)\right) d t\right\} \\
& \geq \lim _{\lambda \rightarrow 0} \inf _{\alpha} \lambda \int_{0}^{\infty} f\left(y_{\alpha}^{k_{0}}(t)\right) d t+C_{2}
\end{aligned}
$$

and thus

$$
d_{f}>\frac{M C_{1}}{C_{2} C_{3}}\left(C_{1}-C_{2}+1\right)+C_{2}
$$

where we used (55), (66).

However, these relationship leads a contradiction to (55) and we have proved the property $\left(A_{0}\right)$. Similarly, in the finite horizon case, we have Vol. $14, \mathrm{n}^{\circ}$ 4-1997. 
on one hand

$$
\begin{aligned}
d_{f} & =\lim _{T \rightarrow \infty} \frac{1}{T} u\left(y_{\alpha}^{1} T\right) \\
& =\lim _{T^{\prime} \rightarrow \infty} \inf _{\alpha}\left\{\frac{1}{T^{\prime}} \int_{0}^{T^{\prime}} f\left(y_{\alpha}^{1}(t)\right)+g\left(y_{\alpha}^{1}(t), \alpha(t)\right) d t\right\} \\
& \leq \varlimsup_{T^{\prime} \rightarrow \infty} \frac{1}{T^{\prime}} \int_{0}^{T^{\prime}} f\left(y_{\alpha_{0}}^{1}(t)\right) d t+C_{1} \\
& =\varlimsup_{T^{\prime} \rightarrow \infty} \frac{1}{T^{\prime}} \int_{0}^{T^{\prime}} f\left(y_{\alpha_{0}}^{1}(t)\right)\left(1-\chi_{\tilde{U}_{k_{0}}}\right)\left(y_{\alpha_{0}}^{1}(t)\right) d t+C_{1}
\end{aligned}
$$

and thus

$$
d_{f} \leq \frac{M C_{1}}{C_{2} C_{3}}\left(C_{1}-C_{2}+1\right)\left(1-\frac{C_{3} C_{3}}{M C_{1}}\right)+C_{1},
$$

where we used $(55),(71),(75),(77),(78)$. On the other hand

$$
d_{f}=\lim _{T \rightarrow \infty} \frac{1}{T} u\left(y^{k_{0}}, T\right) \geq \lim _{T \rightarrow+\infty} \inf _{\alpha} \int_{0}^{T} f\left(y_{\alpha}^{k_{0}}(t)\right) d t+C_{2}
$$

and thus

$$
d_{f} \geq \frac{M C_{1}}{C_{2} C_{3}}\left(C_{1} \cdot C_{2}+1\right)+C_{2},
$$

where we used (55) and (66). Then (81), (82) lead the same contradiction.

Finally, we shall prove the statement of Remark 5.

Proof of Remark 5. - We shall prove it in the following two steps: (i) proof of $Z_{0} \subset \bar{\Omega}_{0}$, (ii) proofs of the invariance and the connectedness of $Z_{0}$. The property $\left(A_{0}^{D}\right)$ comes from $\left(A_{0}\right)$ in Theorem 2 .

(i) Proof of $Z_{0} \subset \bar{\Omega}_{0}$. First, we remark that this assertion is implied by the positive invariance of $\Omega_{0}$ i.e. $T_{t} \Omega_{0} \subset \Omega_{0}$ for all $t \geq 0$. In fact, if we have the above invariance of $\Omega_{0}$ then $\bar{\Omega}_{0}$ is also positive invariant and all trajectories starting from the points in $\Omega_{0}$ stay in $\bar{\Omega}_{0}$, thus by the definition of $Z_{0}$ we see that $Z_{0} \subset \bar{\Omega}_{0}$. To see that $T_{t} \Omega_{0} \subset \Omega_{0}$ for all $t \geq 0$, let $x_{0} \in \Omega_{0}, T_{0} \geq 0$ be arbitrary. Then

$$
\lambda u_{\lambda}\left(x_{0}\right)=\lambda \int_{0}^{T_{0}} e^{-\lambda s} f\left(x_{0}(s)\right) d s+\lambda e^{-\lambda T} u_{\lambda}\left(x_{0}\left(T_{0}\right)\right)
$$


leads $x_{0}\left(T_{0}\right) \in \Omega_{0}$. Similarly, in the finite horizon case

$$
\frac{1}{T} u\left(x_{0}, T\right)=\frac{1}{T} \int_{0}^{T_{0}} f\left(x_{0}(s)\right) d s+\frac{1}{T} u\left(x_{0}\left(T_{0}\right), T-T_{0}\right)
$$

leads $x_{0}\left(T_{0}\right) \in \Omega_{0}$. Therefore, we have proved $Z_{0} \subset \bar{\Omega}_{0}$.

(ii) Proof of the connectedness and the invariance of $Z_{0}$. The invariance (in particular the negative invariance) can be proved similarly as in the proof of Remark 2. To see the connectedness, let $U_{1}, U_{2} \subset \bar{\Omega}$ be two open sets such that $U_{1} \cap U_{2}=\emptyset, U_{1} \cup U_{2} \supset Z_{0}, U_{1} \cap Z_{0} \neq \emptyset, U_{2} \cap Z_{0} \neq \emptyset$. Let $z_{1} \in U_{1} \cap Z_{0}, z_{2} \in U_{2} \cap Z_{0}$ and take $\varepsilon>0$ small enough such that $U_{\varepsilon}\left(z_{1}\right) \subset U_{1}, U_{\varepsilon}\left(z_{2}\right) \subset U_{2}$. By the property $\left(Z_{0}\right)$, since $Z_{0} \subset \bar{\Omega}_{0}$ there exist a point $y \in \Omega_{0} \cap U_{\varepsilon}\left(z_{1}\right)$ and $T_{\varepsilon}>0$ such that $y\left(T_{\varepsilon}\right) \in U_{\varepsilon}\left(z_{2}\right)$. However, since $\left\{y(t) \mid 0 \leq t \leq T_{\varepsilon}\right\}$ is connected it leads a contradiction. Therefore, $Z_{0}$ is connected.

\section{GENERAL REMARK}

Finally, in this section, we shall make a general remark and two questions concerning the ergodic (long time averaged) attractor.

Remark 6. - We have seen that the convergence property (15) (resp. (16)) in Theorem 1 and (21) (resp. (22)) in Theorem 2 implies the existence of the sets $Z$ and $Z_{0}$ respectively, for the controlled system considered in the compact set $\bar{\Omega}$. So, it is natural to ask whether the same is also true for the system in a non-compact set (for example $\Omega=\mathbf{R}^{n}$ or a bounded set in a Banach space (see [9], [16]) etc...). We believe, although we have not yet demonstrated it rigorously, the answer is negative.

Question 1. - Is it possible to precise the behavior of the trajectories on $Z$ ?

Question 2. - Can we have some information on the geometric property of the attractors $Z$ and $Z_{0}$ by the properties $(Z)$ and $\left(Z_{0}\right)$ ?

\section{REFERENCES}

[1] V. I. ARnold and A. Avez, Problèmes ergodiques de la mécanique classique, GauthierVillars, Paris, 1967.

[2] G. Barles and P. L. Lions, Fully nonlinear Neumann type boundary conditions for firstorder Hamilton-Jacobi equations, Nonlinear Anal. Theory Methods Appl., Vol. 16, 1991, pp. 143-153.

Vol. $14, n^{\circ} 4-1997$. 
[3] I. Capuzzo-Dolcetta and M. G. Garron, Oblique derivative problems and invariant measures, Ann. Scuola Norm. Sup. Pisa, Vol. 23, 1986. pp. 689-720.

[4] I. Capuzzo-DolcetTa and P. L. Lions, Hamilton-Jacobi equations with state constraints, Trans. Amer. Math. Soc., Vol. 318, 1990, pp. 643-683.

[5] I. Capuzzo-DolcetTa and J. L. MENALDI, On the deterministic optimal stopping time problem in the ergodic case. Theory and applications of nonlinear control system. North Holland, 1986, pp. 453-460.

[6] I. P. Cornfei D, S. V. Fomin and Ya. G. Sinai, Ergodic: Theory, New York, Springer-Verlag, 1982.

[7] M. G. Crandall and P. L. Lions, Viscosity solutions of Hamilion-Jacobi equations, Trans. Amer. Math. Soc., Vol. 277, 1983, pp. 1-42.

[8] P. Dupuls and H. IsHi, On oblique derivative problems for fully nonlinear second-order elliptic partial differential equations on non smooth domains, Nonlinear Anal. Theory Methods Appl., Vol. 15, 1990, pp. 1123-1138.

[9] J. GUCKENHEIMER and P. Holmes, Nonlinear oscillations, dynamical systems and bifurcations of vector fields, Springer-Verlag, $3^{\text {rd }}$ edition, 1990.

[10] P.L. Lions, Generalized solutions of Hamilton-Jacobi equations, Research Notes in Mathematics, Vol. 69, Pitman, Boston, MA, 1982.

[11] P. L. Lions, Neumann type boundary conditions for Hamilmton-Jacobi equations, Duke J. Math., Vol. 52, 1985, pp. 793-820.

[12] P. L. Lions and B. Perthame, Quasi-variational inequalities and ergodic impulse control. SIAM J. Control and Optimization, Vol. 24, 1986, pp. 604-615.

[13] M. RoBIN, On some impulse control problems with lon run average control, SIAM J. Control and Optimization, Vol. 19, 1981, pp. 333-358.

[14] B. Simon, Functional integration and quantum physics, Academic Press, 1979.

[15] II. M. SONER, Optimal control with state-space constraint I, SIAM J. Control Optim., Vol. 24, 1986, pp. 552-562; Optimal control with state-space constraint II, SIAM J. Control Optim., Vol. 24, 1986, pp. 1110-1122.

[16] R. TEMAM, Infinite-dimensional dynamical systems in mecanics and physics, SpringerVerlag, $[988$

(Manuscript received May 29, 1995.) 